\title{
DENSITY OF STATES FOR PLATONIC CRYSTALS AND CLUSTERS*
}

\author{
M. J. A. SMITH ${ }^{\dagger}$, M. H. MEYLAN ${ }^{\ddagger}$ AND R. C. MCPHEDRAN $\S$
}

\begin{abstract}
The density of states, which measures the density of the spectrum, is evaluated for a platonic crystal (periodically structured elastic plate) using the Green's function approach. Results are presented not only for the standard density of states, but also for the mutual, local, and spectral density of states. These other state functions provide a pathway to the standard density of states and characterize the radiative and other properties of the crystal. This is the first known examination of the density of states for a platonic crystal and extends the existing Green's function approach for photonic crystals to thin, elastic plates. As a motivating example the theory is applied to the problem of a square array of pins embedded in a thin plate. The density of states functions for an empty lattice (a uniform plate) are also presented in order to give a clear illustration of the steps in the derivation. Careful numerical calculations are given which reveal the complex behavior of the crystal, including intervals of suppressed density of states. These results are compared to calculations for a finite crystal with an interior source, and the behaviors of the finite and infinite systems are shown to be connected through the density of states.
\end{abstract}

Key words. platonic crystal, density of states, sources, flexural waves, Kirchoff-Love plate, field localization, finite crystals

AMS subject classifications. 31A30, 35J08, 35J40, 35Q99, 47A40

DOI. $10.1137 / 130950501$

1. Introduction. In the majority of the photonic, phononic, and platonic crystal literature, plane waves or localized beams are used as the primary tool to examine the behavior of waves inside a crystal; in contrast, the behavior of sources has received considerably less attention. This is largely due to the difficulty in computing the density of states (DOS) functions. These functions characterize the behavior of sources placed inside an array, and are measures of the density of the spectrum [15]. It is important to understand how different incident waves behave in response to periodic structures, because powerful techniques have recently evolved for guiding waves and coupling them to sources [12]. This is especially true for the design of metamaterials which are used for guiding light, sound, and elastic vibrations through periodic structures.

While various methods have been developed to calculate the DOS and LDOS (local density of states), including the Bloch method [14], using transfer matrices (for a one-dimensional crystal), or via multiple scattering theory $[38,21]$, here we use the Green's function approach to obtain the necessary DOS functions [17]. This method is advantageous because we are able to obtain compact analytical expressions for the

* Received by the editors December 23, 2013; accepted for publication (in revised form) June 11, 2014; published electronically October 2, 2014.

http://www.siam.org/journals/siap/74-5/95050.html

${ }^{\dagger}$ Aix-Marseille Université, CNRS, Centrale Marseille, Institut Fresnel, 13013 Marseille, France (mike.smith@fresnel.fr).

${ }^{\ddagger}$ School of Mathematical and Physical Sciences, The University of Newcastle, New South Wales 2308, Newcastle, Australia (mike.meylan@newcastle.edu.au).

$\S$ CUDOS ARC Centre of Excellence, School of Physics, University of Sydney, New South Wales 2006, Sydney, Australia, and Department of Mathematical Sciences, M \& O Building, Peach Street, Liverpool L69 3BX, UK (ross@physics.usyd.edu.au). The research of this author was supported by a Marie Curie Intra-European Fellowship and by the Australian Research Council through its Discovery Grant Scheme. The Centre for Ultrahighbandwidth Devices for Optical Systems (CUDOS) is an ARC Centre of Excellence (Project CE110001018). 


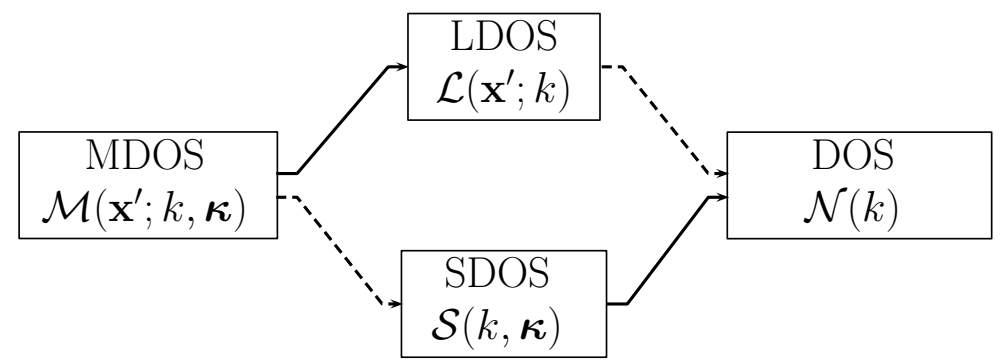

FIG. 1. An outline of the relationship between the different density of state functions, where broken lines denote integration over the WSC and solid lines represent integration over the BZ.

DOS functions, which are straightforward to evaluate numerically, once the dispersion surfaces are known. In [17], it was shown that there are multiple routes for deriving the DOS, and that ideally one should begin by evaluating the most general DOS function, which is the mutual DOS (MDOS). From this function one can then obtain the spectral DOS (SDOS), the local DOS (LDOS), and ultimately, the DOS. Each of these density functions corresponds to a different problem physically, but they are ultimately related through integration over reciprocal and real space. That is, by integrating the MDOS over the Wigner-Seitz cell (WSC), one can obtain the SDOS, or by integrating the MDOS over the Brillouin zone (BZ), one can obtain the LDOS. Furthermore, by integrating the LDOS over the WSC, or the SDOS over the BZ, we arrive at the DOS. This is characterized in Figure 1.

The LDOS function is the easiest to physically understand, as it is proportional to the total power radiated by a source through an array [34]. It depends on the location of a single source placed inside an infinitely large crystal and on the frequency at which it oscillates. However, mathematically deriving the Green's function for a single source directly (and thus the LDOS) can be difficult. It is because of this that we compute the Green's function for the MDOS problem first. Physically the MDOS problem corresponds to a periodic structure of infinite extent, where for each scatterer we have an associated source. Mathematically deriving the Green's function for this problem is much simpler than deriving the single-source Green's function, and it is through this MDOS Green's function that all the other functions are obtained here. This is simpler because Bloch-Floquet theory can be used on both the array of sources and the array of inclusions to reduce the problem to a single cell. The LDOS can also be regarded as a measure of how well a source can couple to the band surfaces, and it is also proportional to the spontaneous emission rate of atoms in a crystal [25, 34]. Similarly, the SDOS is a function which describes the radiation pattern of a phased array (or directionality of the waves through an array) [25] and can be regarded as a proxy for the dispersion relation. Both the LDOS and SDOS are relevant functions to consider for any crystal problem, as photonic and phononic crystals redistribute DOS in both frequency and real space [10]. One of the most powerful uses of the DOS is to reveal the location of complete band gaps and the frequencies at which the critical points of band surfaces are located [9].

There has been a considerable amount of work done on the LDOS for finite photonic crystals. This includes the manipulation of spontaneous emission in photonic crystal fibers [25] and the localization of energy by suppression of the DOS [10]. Finite clusters of suitably large size have also been shown to provide an efficient approximation for the DOS of an infinite crystal [2]. Additionally, [1] shows that 
perfectly square clusters exhibit energy leakage at the corners. This can be suppressed by changing the geometry slightly; however, this diminishes frequency shifts and peak LDOS values.

Here we investigate the dynamics of a thin elastic plate of infinite extent, which is periodically pinned at each point in a square array throughout the domain. Such structures involving the biharmonic plate equation are typically referred to as platonic crystals (PlaCs) [18]. Pinned PlaCs have been shown to exhibit many novel diffraction, refraction, and other phenomena, including negative refraction [29], strongly localized defect modes [27], and Dirac cone dispersion in the center of the BZ [30]. Additionally, platonic designs exhibiting ultrahigh $Q$-factor trapping have been demonstrated using gratings of pinned and circular inclusions [11]. Other interesting nonpinned structures include platonic absorbers [4] and platonic cloaks [7,33]. The problem considered here is the first investigation into the DOS for thin elastic plates, and it is not obvious that properties of the DOS for the Helmholtz equation extend straightforwardly to the biharmonic equation, given that it is a higher-order problem (fourth-order equation) and the Green's function is regular at the origin for the plate. Recently, an investigation into the forcing of the central pin in a rectangular pinned lattice was made by [31], in a similar vein to work on waves through lattices by [16]. In [31] they examined the far-field wave behavior of a forced pin through a lattice, which is defined by integration over the isofrequency contours at constant frequency. This bears resemblance to the LDOS problem considered here, but it is ultimately related to a different physical problem (here there is no central defect).

In this work, we demonstrate that pinned PlaCs feature intervals in $k$-space where the DOS is suppressed, due to the curvature of the associated band surfaces. Using finite PlaCs, it is shown that these regions of suppressed DOS correspond to strong field concentrations in the displacement, despite being in-band. These field concentrations arise because the source is unable to perfectly couple to the available bands, resulting in semi-localized modes. These do not correspond to a resonance of the finite structure, as the amplitude of the field does not grow as the cluster size is increased.

Also presented here are LDOS surfaces at a selection of wave numbers $k$, where it can be observed that the position of the source significantly changes the power radiated through the array. The source coordinate for optimal power radiation does not remain fixed as $k$ is increased, but varies significantly. We also compute the LDOS as a function of $k$ for different source locations. When the source is located at the corner of the WSC we observe wide intervals of zero resonances (corresponding to strong field confinement), which occurs over a wider frequency range than the interval of suppressed DOS. This shows that field localization depends strongly on both the source location and wave number. Finally we demonstrate that, within a region of suppressed DOS, the emission pattern of a finite cluster can be manipulated by a careful choice of source location.

An overview of the paper is as follows. In section 2 we compute the quasi-periodic Green's function for an elastic plate in general, which is then used to compute the DOS functions for an empty lattice in section 3. After considering this motivating example, we consider the Green's function and DOS functions for a pinned plate in section 4 , as well as the LDOS and DOS functions for a finite cluster of pins in section 5 . Results are then given in section 6 , which is followed by concluding remarks in the final section. 
2. Green's functions for the biharmonic plate equation. The governing equation for a thin, elastic plate in the frequency domain is given by

$$
\left(\Delta^{2}-k^{4}\right) w(\mathbf{x})=0,
$$

where $\Delta=\partial_{x}^{2}+\partial_{y}^{2}$ represents the two-dimensional Laplacian, $k^{2}=\omega \sqrt{\rho h / D}$, and $w(\mathbf{x})$ is the displacement. Here $k$ denotes the nondimensionalized wave number, $\omega$ the angular frequency, and $\rho, D$, and $h$ are the parameters for the plate. A time-harmonic wave dependence of $\exp (-\mathrm{i} \omega t)$ is assumed, and we nondimensionalize the problem so that $\sqrt{\rho h / D}=1$. Furthermore, we assume that the plate is pinned at an infinite array of points,

$$
w\left(\mathbf{R}_{p}\right)=0,
$$

where

$$
\mathbf{R}_{p}=m\left(\begin{array}{c}
d \\
0
\end{array}\right)+n\left(\begin{array}{l}
0 \\
d
\end{array}\right)
$$

denotes the array vector for a square lattice of period $d$, and $m, n \in \mathbb{Z}$. We define the quasi-periodic Green's function for a phased array of sources, which satisfies

$$
\left(\Delta^{2}-k^{4}\right) G_{M}\left(\mathbf{x}, \mathbf{x}^{\prime} ; k, \boldsymbol{\kappa}\right)=\sum_{p} \delta\left(\mathbf{x}-\mathbf{x}^{\prime}-\mathbf{R}_{p}\right) \mathrm{e}^{\mathrm{i} \boldsymbol{\kappa} \cdot \mathbf{R}_{p}} .
$$

For a given Bloch vector $\boldsymbol{\kappa}$ there exist Bloch states $\psi_{l}$ with corresponding wave numbers $k_{l}$ which satisfy

$$
\Delta^{2} \psi_{l}=k_{l}^{4} \psi_{l}
$$

where $l$ denotes the $l$ th band surface and the Bloch states are normalized by integration over the WSC:

$$
\int_{\mathrm{WSC}} \psi_{l}^{*}(\mathbf{x} ; k, \boldsymbol{\kappa}) \psi_{m}(\mathbf{x} ; k, \boldsymbol{\kappa}) \mathrm{d} \mathbf{x}=\delta_{l m} .
$$

For a two-dimensional square array the WSC is given by $\mathbf{x} \in[-d / 2, d / 2] \times[-d / 2, d / 2]$. We can write the quasi-periodic Green's function in the form

$$
G_{M}\left(\mathbf{x}, \mathbf{x}^{\prime} ; k, \boldsymbol{\kappa}\right)=\sum_{m} \frac{\psi_{m}^{*}\left(\mathbf{x}^{\prime} ; k, \boldsymbol{\kappa}\right) \psi_{m}(\mathbf{x} ; k, \boldsymbol{\kappa})}{k_{m}^{4}-k^{4}} .
$$

It is then advantageous to decompose this representation into real and imaginary components. This can be done easily for the Helmholtz equation using the Plemelj formula [24], a result which arises from using the half-residue theorem along the real line. For the biharmonic equation we take the more general approach and factorize the denominator of (2.7) to obtain

$$
\frac{1}{k_{m}^{4}-k^{4}}=\frac{-1}{\left(k-k_{m}\right)\left(k+k_{m}\right)\left(k^{2}+k_{m}^{2}\right)} \text {. }
$$

The residue at $k=k_{m}$ of $(2.8)$ is $1 /\left(4 k_{m}^{3}\right)$, and since the imaginary part of $G_{M}$ has a semi-residue contribution, one can write the Green's function in the form

$$
G_{M}\left(\mathbf{x}, \mathbf{x}^{\prime} ; k, \boldsymbol{\kappa}\right)=\mathrm{P}\left[G_{M}\right]+\frac{\mathrm{i} \pi}{4 k^{3}} \sum_{m} \psi_{m}^{*}\left(\mathbf{x}^{\prime} ; \boldsymbol{\kappa}\right) \psi_{m}(\mathbf{x} ; \boldsymbol{\kappa}) \delta\left(k-k_{m}\right),
$$

Copyright (C) by SIAM. Unauthorized reproduction of this article is prohibited. 
where $\mathrm{P}\left[G_{M}\right]$ denotes the Cauchy principal value integral of $G_{M}$ along the real line. Note that the sign of the imaginary component of $G_{M}$ changes depending on whether the imaginary part added to $k \in \mathbb{R}^{+}$is positive or negative [19]. It is the imaginary part of the Green's function which possesses the information required to obtain the DOS functions [13].

3. The quasi-periodic Green's function for an empty lattice. Before calculating the various DOS functions for the pinned plate we consider the empty lattice problem in order to illustrate the method. In this case the Green's function satisfies (2.4), where the Dirac delta functions are taken to have positive amplitude and the $E$ superscript is used to denote the empty lattice problem. The sign of the delta function corresponds to a positive DOS for a thin plate as $\left|\mathbf{x}-\mathbf{x}^{\prime}\right| \rightarrow \infty$ [6]. This is in contrast to the Helmholtz operator, where the delta function has a negative amplitude. Following from the representation (2.7), one can use partial fractions and decompose the Green's function to obtain

$$
G_{M}^{E}\left(\mathbf{x}, \mathbf{x}^{\prime} ; k, \boldsymbol{\kappa}\right)=\left(\frac{1}{2 k^{2}}\right) \sum_{n}\left(\frac{\psi_{n}^{E}(\mathbf{x} ; \boldsymbol{\kappa})\left[\psi_{n}^{E}\left(\mathbf{x}^{\prime} ; \boldsymbol{\kappa}\right)\right]^{*}}{k^{2}+k_{n}^{2}}-\frac{\psi_{n}^{E}(\mathbf{x} ; \boldsymbol{\kappa})\left[\psi_{n}^{E}\left(\mathbf{x}^{\prime} ; \boldsymbol{\kappa}\right)\right]^{*}}{k^{2}-k_{n}^{2}}\right),
$$

where $\psi_{n}^{E}$ denotes the Bloch mode for an empty lattice with corresponding wave number $k_{n}=\left|\mathbf{Q}_{n}\right|$. Comparing this to the multipole representation for the quasiperiodic Green's function satisfying (2.4), we can then establish the form of the Bloch modes. After reversing the order of the terms, this is given by (see [23])

$$
\begin{aligned}
G_{M}^{E}\left(\mathbf{x}, \mathbf{x}^{\prime} ; k, \boldsymbol{\kappa}\right) & =\sum_{p} \frac{\mathrm{i}}{8 k^{2}}\left[H_{0}^{(1)}\left(k\left|\mathbf{x}-\mathbf{x}^{\prime}-\mathbf{R}_{p}\right|\right)-\frac{2}{\pi \mathrm{i}} K_{0}\left(k\left|\mathbf{x}-\mathbf{x}^{\prime}-\mathbf{R}_{p}\right|\right)\right] \mathrm{e}^{\mathrm{i} \boldsymbol{\kappa} \cdot \mathbf{R}_{p}} \\
& =\left(\frac{1}{2 k^{2}}\right)\left[G^{\mathrm{H}}\left(\mathbf{x}, \mathbf{x}^{\prime} ; k, \boldsymbol{\kappa}\right)-G^{\mathrm{M}}\left(\mathbf{x}, \mathbf{x}^{\prime} ; k, \boldsymbol{\kappa}\right)\right],
\end{aligned}
$$

where one can use the plane wave representations

$$
\begin{aligned}
G^{\mathrm{H}}\left(\mathbf{x}, \mathbf{x}^{\prime} ; k, \boldsymbol{\kappa}\right) & =-\frac{1}{A_{\mathrm{WSC}}} \sum_{h} \frac{\mathrm{e}^{\mathrm{i} \mathbf{Q}_{h} \cdot\left(\mathbf{x}-\mathbf{x}^{\prime}\right)}}{k^{2}-\left|\mathbf{Q}_{h}\right|^{2}}, \\
G^{\mathrm{M}}\left(\mathbf{x}, \mathbf{x}^{\prime} ; k, \boldsymbol{\kappa}\right) & =\frac{1}{A_{\mathrm{WSC}}} \sum_{h} \frac{\mathrm{e}^{\mathrm{i} \mathbf{Q}_{h} \cdot\left(\mathbf{x}-\mathbf{x}^{\prime}\right)}}{k^{2}+\left|\mathbf{Q}_{h}\right|^{2}},
\end{aligned}
$$

to determine that the Bloch modes for an empty lattice are given by

$$
\psi_{h}^{E}(\mathbf{x} ; \boldsymbol{\kappa})=\frac{1}{\sqrt{A_{\mathrm{WSC}}}} \mathrm{e}^{\mathrm{i} \mathbf{Q}_{h} \cdot \mathbf{x}} .
$$

This is identical to the Bloch mode corresponding to the Helmholtz equation in an empty lattice (an outline of this problem is given in the supplementary material).

3.1. Density of states for an empty lattice. Having previously determined the form of the Bloch modes $\psi_{h}$, we can then compute the MDOS for an empty lattice. This is given by

$$
\mathcal{M}\left(\mathbf{x}^{\prime} ; k, \boldsymbol{\kappa}\right)=\frac{4 k^{3}}{\pi} \operatorname{Im}\left(G_{M}^{E}\left(\mathbf{x}^{\prime}, \mathbf{x}^{\prime} ; k, \boldsymbol{\kappa}\right)\right)=\frac{1}{A_{\mathrm{WSC}}} \sum_{h} \delta\left(k-\left|\mathbf{Q}_{h}\right|\right),
$$


where the MDOS is scaled in order to balance the semiresidue contribution in (2.9), in a vein similar to [17] for the Helmholtz equation. From its definition, the SDOS can then be obtained via integration over the WSC to obtain

$$
\mathcal{S}(k, \boldsymbol{\kappa})=\frac{1}{A_{\mathrm{WSC}}} \int_{\mathrm{WSC}} \mathcal{M}\left(\mathbf{x}^{\prime} ; k, \boldsymbol{\kappa}\right) \mathrm{d} \mathbf{x}^{\prime}=\mathcal{M}\left(\mathbf{x}^{\prime} ; k, \boldsymbol{\kappa}\right),
$$

since there is no $\mathbf{x}^{\prime}$ dependence. Furthermore the LDOS can be determined from the MDOS via integration over the BZ [17], and we can see that for the empty lattice

$$
\mathcal{L}\left(\mathbf{x}^{\prime} ; k\right)=\frac{1}{A_{\mathrm{BZ}}} \int_{\mathrm{BZ}} \mathcal{M}\left(\mathbf{x}^{\prime} ; k, \boldsymbol{\kappa}\right) \mathrm{d} \boldsymbol{\kappa}=\frac{1}{A_{\mathrm{BZ}}} \int_{\mathrm{BZ}} \mathcal{S}(k, \boldsymbol{\kappa}) \mathrm{d} \boldsymbol{\kappa}=\mathcal{N}(k),
$$

where the BZ is given by $\kappa \in[-\pi / d, \pi / d] \times[-\pi / d, \pi / d]$ for a two-dimensional square array. This can be evaluated further, and so we write

$$
\mathcal{N}(k)=\frac{1}{A_{\mathrm{BZ}}} \int_{\mathrm{BZ}} \mathcal{S}(k, \boldsymbol{\kappa}) \mathrm{d} \boldsymbol{\kappa}=\frac{1}{A_{\mathrm{WSC}} A_{\mathrm{BZ}}} \int_{\mathrm{BZ}} \sum_{h} \delta\left(k-\left|\mathbf{Q}_{h}\right|\right) \mathrm{d} \boldsymbol{\kappa},
$$

where $A_{\mathrm{WSC}} A_{\mathrm{BZ}}=(2 \pi)^{2}$ for a square lattice, and via the change of variables

$$
\boldsymbol{Q}_{h}=\boldsymbol{\kappa}+\mathbf{K}_{h}=\left(\rho_{h} \sin \phi_{h}, \rho_{h} \cos \phi_{h}\right),
$$

one can obtain the result

$$
\int_{\mathrm{BZ}} \sum_{h} \delta\left(k-\left|\mathbf{Q}_{h}\right|\right) \mathrm{d} \boldsymbol{\kappa}=\sum_{h} \iint \delta\left(k-\rho_{h}\right) \rho_{h} \mathrm{~d} \rho_{h} \mathrm{~d} \phi_{h}=2 \pi k .
$$

Subsequently the DOS can be expressed in the form

$$
\mathcal{N}(k)=\frac{1}{A_{\mathrm{WSC}} A_{\mathrm{BZ}}}(2 \pi k)=\frac{k}{2 \pi} .
$$

In summary, we have $\mathcal{M}=\mathcal{S}$ and $\mathcal{N}=\mathcal{L}=k /(2 \pi)$ for an empty lattice of sources in a thin plate.

3.2. LDOS in an empty lattice. Alternatively, we can derive an identical result by using the free-space Green's function (for a single source), which satisfies

$$
\left(\Delta^{2}-k^{4}\right) G_{L}^{E}\left(\mathbf{x}, \mathbf{x}^{\prime} ; k\right)=\delta\left(\left|\mathbf{x}-\mathbf{x}^{\prime}\right|\right)
$$

and is given in closed form by (see [26])

$$
G_{L}^{E}\left(\mathbf{x}, \mathbf{x}^{\prime} ; k\right)=\frac{\mathrm{i}}{8 k^{2}}\left[H_{0}^{(1)}\left(k\left|\mathbf{x}-\mathbf{x}^{\prime}\right|\right)-\frac{2}{\pi \mathrm{i}} K_{0}\left(k\left|\mathbf{x}-\mathbf{x}^{\prime}\right|\right)\right] .
$$

From (3.7) we can then show that

$$
\mathcal{L}\left(\mathbf{x}^{\prime} ; k\right)=\frac{4 k^{3}}{\pi A_{\mathrm{BZ}}} \operatorname{Im}\left\{\int_{\mathrm{BZ}} G_{M}\left(\mathbf{x}^{\prime}, \mathbf{x}^{\prime} ; k, \boldsymbol{\kappa}\right) \mathrm{d} \boldsymbol{\kappa}\right\}=\frac{4 k^{3}}{\pi} \operatorname{Im}\left\{G_{L}\left(\mathbf{x}, \mathbf{x}^{\prime} ; k\right)\right\}=\frac{k}{2 \pi},
$$

and that

$$
\mathcal{N}(k)=\frac{1}{A_{\mathrm{WSC}}} \int_{\mathrm{WSC}} \mathcal{L}\left(\mathbf{x}^{\prime} ; k\right) \mathrm{d} \mathbf{x}^{\prime}=\mathcal{L}\left(\mathbf{x}^{\prime} ; k\right)=\frac{k}{2 \pi},
$$

Copyright $@$ by SIAM. Unauthorized reproduction of this article is prohibited. 
since there is no $\mathbf{x}^{\prime}$ dependence. Note that it is also possible to obtain the Green's function for the LDOS problem via integration:

$$
G_{L}\left(\mathbf{x}, \mathbf{x}^{\prime} ; k\right)=\frac{1}{A_{\mathrm{BZ}}} \int_{\mathrm{BZ}} G_{M}\left(\mathbf{x}, \mathbf{x}^{\prime} ; k, \boldsymbol{\kappa}\right) \mathrm{d} \boldsymbol{\kappa} .
$$

Having considered the case of the empty lattice, we now proceed to the problem of computing the DOS functions for an elastic plate which possesses a doubly periodic array of pins.

4. The pinned elastic plate problem. We consider a thin elastic plate which satisfies the biharmonic plate equation (2.5), where the displacement is taken to be zero at each point in a two-dimensional lattice, as shown in (2.2).

4.1. Multipole representation of the MDOS Green's function. Using the method of fictitious sources [37], it is possible to represent the MDOS Green's function $G_{M}\left(\mathbf{x}, \mathbf{x}^{\prime} ; k, \boldsymbol{\kappa}\right)$ in the form

$$
G_{M}^{P}\left(\mathbf{x}, \mathbf{x}^{\prime} ; k, \boldsymbol{\kappa}\right)=G_{M}^{E}\left(\mathbf{x}, \mathbf{x}^{\prime} ; k, \boldsymbol{\kappa}\right)+a_{00} G_{M}^{E}(\mathbf{x}, \mathbf{0} ; k, \boldsymbol{\kappa}),
$$

where the first term on the right-hand side represents the array of sources, and the second term denotes the contribution from the pins. There is only one unknown $a_{00}$, as the quasi periodicity of both the array and the pinned plate are encapsulated by the Green's function $G_{E}^{M}$ defined in (3.2). However, the representation (3.2) is only conditionally convergent and requires acceleration in order to be evaluated numerically. This is achieved here using lattice sums [5, 23, 22], and they admit the representations

$$
\begin{aligned}
G^{\mathrm{H}}\left(\mathbf{x}, \mathbf{x}^{\prime}\right) & =\frac{\mathrm{i}}{4} H_{0}^{(1)}(k|\boldsymbol{\xi}|)+\frac{\mathrm{i}}{4} \sum_{l=-\infty}^{\infty} S_{l}^{\mathrm{H}} J_{l}(k|\boldsymbol{\xi}|) \mathrm{e}^{-\mathrm{i} l \arg \boldsymbol{\xi}} \\
G^{\mathrm{M}}\left(\mathbf{x}, \mathbf{x}^{\prime}\right) & =\frac{1}{2 \pi} K_{0}(k|\boldsymbol{\xi}|)+\frac{1}{2 \pi} \sum_{l=-\infty}^{\infty} S_{l}^{\mathrm{K}} I_{l}(k|\boldsymbol{\xi}|) \mathrm{e}^{-\mathrm{i} l \arg \boldsymbol{\xi}}
\end{aligned}
$$

where $\boldsymbol{\xi}=\mathbf{x}-\mathbf{x}^{\prime}$ and $\phi_{p}=\arg \mathbf{R}_{p}$. Convergent representations for the array sums $S_{l}^{\mathrm{H}}$ and $S_{l}^{\mathrm{K}}$ can be found in $[22,5]$ for the case of a square lattice and are given by $S_{l}^{\mathrm{H}}(k, \boldsymbol{\kappa})=-\delta_{l 0}+\mathrm{i} S_{l}^{\mathrm{Y}}(k, \boldsymbol{\kappa})$,

$$
\begin{aligned}
S_{l}^{\mathrm{Y}}(k, \boldsymbol{\kappa})=\frac{1}{J_{l+3}(k|\boldsymbol{\eta}|)}(- & {\left[Y_{3}(k|\boldsymbol{\eta}|)+\frac{1}{\pi} \sum_{n=1}^{3} \frac{(3-n) !}{(n-1) !}\left(\frac{2}{k|\boldsymbol{\eta}|}\right)^{5-2 n}\right] \delta_{l, 0} } \\
& \left.-\frac{4 \mathrm{i}^{l}}{d^{2}} \sum_{p}\left(\frac{k}{\left|\mathbf{Q}_{p}\right|}\right)^{3} \frac{J_{l+3}\left(\left|\mathbf{Q}_{p}\right||\boldsymbol{\eta}|\right)}{\left|\mathbf{Q}_{p}\right|^{2}-k^{2}} \mathrm{e}^{\mathrm{i} l \theta_{p}}\right)
\end{aligned}
$$

and

$$
\begin{aligned}
S_{l}^{\mathrm{K}}(k, \boldsymbol{\kappa})=\frac{1}{I_{l+3}(k|\boldsymbol{\eta}|)}\left(\left[K_{3}(k|\boldsymbol{\eta}|)\right.\right. & \left.-\frac{8}{(k|\boldsymbol{\eta}|)^{3}}+\frac{1}{k|\boldsymbol{\eta}|}-\frac{k|\boldsymbol{\eta}|}{8}\right] \delta_{l, 0} \\
+ & \left.\frac{2 \pi \mathrm{i}^{l}}{d^{2}} \sum_{p}\left(\frac{k}{\left|\mathbf{Q}_{p}\right|}\right)^{3} \frac{J_{l+3}\left(\left|\mathbf{Q}_{p}\right||\boldsymbol{\eta}|\right) \mathrm{e}^{\mathrm{i} l \theta_{p}}}{\left|\mathbf{Q}_{p}\right|^{2}+k^{2}}\right),
\end{aligned}
$$

where $\delta_{m n}$ is the Kronecker delta, $\theta_{p}=\arg \mathbf{Q}_{p}, \mathbf{Q}_{p}=\left(\kappa_{x}+2 \pi m / d, \kappa_{y}+2 \pi n / d\right)$, and the vector $\boldsymbol{\eta}$ represents an arbitrary vector positioned inside the BZ.

Copyright $@$ by SIAM. Unauthorized reproduction of this article is prohibited. 
Accordingly we can then impose the pin condition at the origin to obtain

$$
G_{M}^{P}\left(\mathbf{x}, \mathbf{x}^{\prime} ; k, \boldsymbol{\kappa}\right)=G_{M}^{E}\left(\mathbf{x}, \mathbf{x}^{\prime} ; k, \boldsymbol{\kappa}\right)-\frac{G_{M}^{E}\left(\mathbf{0}, \mathbf{x}^{\prime} ; k, \boldsymbol{\kappa}\right) G_{M}^{E}(\mathbf{x}, \mathbf{0} ; k, \boldsymbol{\kappa})}{G_{M}^{E}(\mathbf{0}, \mathbf{0} ; k, \boldsymbol{\kappa})},
$$

where

$$
\begin{aligned}
& G_{M}^{E}(\mathbf{0}, \mathbf{0} ; k, \boldsymbol{\kappa})=G_{M}^{E}\left(\mathbf{x}^{\prime}, \mathbf{x}^{\prime} ; k, \boldsymbol{\kappa}\right), \\
& G_{M}^{E}(\mathbf{x}, \mathbf{0} ; k, \boldsymbol{\kappa})=G_{M}^{E}(\mathbf{0}, \mathbf{x} ; k, \boldsymbol{\kappa})^{*},
\end{aligned}
$$

and

$$
G_{M}^{E}(\mathbf{0}, \mathbf{0} ; k, \boldsymbol{\kappa})=\frac{-1}{8 k^{2}}\left(S_{0}^{\mathrm{Y}}(k, \boldsymbol{\kappa})+\frac{2}{\pi} S_{0}^{\mathrm{K}}(k, \boldsymbol{\kappa})\right) .
$$

If one takes the limit as $\mathbf{x} \rightarrow \mathbf{x}^{\prime}$ in (4.4), using the identities above, this becomes

$$
G_{M}^{P}\left(\mathbf{x}^{\prime}, \mathbf{x}^{\prime} ; k, \boldsymbol{\kappa}\right)=\frac{\left|G_{M}^{E}(\mathbf{0}, \mathbf{0} ; k, \boldsymbol{\kappa})\right|^{2}-\left|G_{M}^{E}\left(\mathbf{x}^{\prime}, \mathbf{0} ; k, \boldsymbol{\kappa}\right)\right|^{2}}{G_{M}^{E}(\mathbf{0}, \mathbf{0} ; k, \boldsymbol{\kappa})} .
$$

For compactness we now define $S(k, \boldsymbol{\kappa})=G_{M}^{E}(\mathbf{0}, \mathbf{0} ; k, \boldsymbol{\kappa})$, which coincides with the dispersion relation for a pinned plate [22]. Note that the denominator of (4.6) is singular at $k=k_{m}$ such that $S\left(k_{m}, \boldsymbol{\kappa}\right)=0$, and so we can deduce that this expression is proportional to the imaginary part of the Green's function. Accordingly we can write

$$
\left|\psi_{l}^{P}\left(\mathbf{x}^{\prime} ; k, \boldsymbol{\kappa}\right)\right|^{2} \propto\left|G_{M}^{E}\left(\mathbf{x}^{\prime}, \mathbf{0} ; k_{l}, \boldsymbol{\kappa}\right)\right|^{2}-\left|G_{M}^{E}\left(\mathbf{0}, \mathbf{0} ; k_{l}, \boldsymbol{\kappa}\right)\right|^{2} .
$$

We now define the nonnormalized form of the Bloch mode by

$$
\left|\phi_{l}^{P}\left(\mathbf{x}^{\prime} ; k, \boldsymbol{\kappa}\right)\right|^{2}=\left|G_{M}^{E}\left(\mathbf{x}^{\prime}, \mathbf{0} ; k_{l}, \boldsymbol{\kappa}\right)\right|^{2}-\left|G_{M}^{E}\left(\mathbf{0}, \mathbf{0} ; k_{l}, \boldsymbol{\kappa}\right)\right|^{2},
$$

where $\left|\psi_{l}^{P}\right|^{2}=\left|A_{l}\right|^{2}\left|\phi_{l}^{P}\right|^{2}$, and scale this to satisfy

$$
\int_{\mathrm{WSC}}\left|\psi_{l}^{P}\right|^{2} \mathrm{~d} \mathbf{x}^{\prime}=\int_{\mathrm{WSC}}\left|A_{l}\right|^{2}\left|\phi_{l}^{P}\right|^{2} \mathrm{~d} \mathbf{x}^{\prime}=1 .
$$

Note that the second Green's function term in (4.8) embodies the dispersion relation; however, it is necessary to include this term in order to ensure that the pin condition is satisfied at $k$-values where band surfaces and light lines intersect.

4.2. Evaluation of the DOS for a pinned elastic plate. Having obtained the form of the Bloch modes, we return to the computation of the MDOS. From the definition of the MDOS in [17] we have

$$
\mathcal{M}\left(\mathbf{x}^{\prime} ; k, \boldsymbol{\kappa}\right)=\frac{4 k^{3}}{\pi} \operatorname{Im}\left(G_{M}^{P}\left(\mathbf{x}^{\prime}, \mathbf{x}^{\prime} ; k, \boldsymbol{\kappa}\right)\right)=\sum_{m}\left|\psi_{m}^{P}\left(\mathbf{x}^{\prime} ; k, \boldsymbol{\kappa}\right)\right|^{2} \delta\left(k-k_{m}\right) .
$$

Accordingly, one then obtains the SDOS via integration of (4.10) over the WSC:

$$
\mathcal{S}(k, \boldsymbol{\kappa})=\frac{1}{A_{\mathrm{WSC}}} \sum_{m} \delta\left(k-k_{m}(\boldsymbol{\kappa})\right)
$$

Copyright (c) by SIAM. Unauthorized reproduction of this article is prohibited. 
(a visualization of the SDOS is provided in the supplementary material). From this we can then evaluate the DOS via the identity

$$
\mathcal{N}(k)=\frac{1}{A_{\mathrm{BZ}}} \int_{\mathrm{BZ}} \mathcal{S}(k, \boldsymbol{\kappa}) \mathrm{d} \boldsymbol{\kappa}=\frac{1}{A_{\mathrm{WSC}} A_{\mathrm{BZ}}} \int_{\mathrm{BZ}} \sum_{m} \delta\left(k-k_{m}(\boldsymbol{\kappa})\right) \mathrm{d} \boldsymbol{\kappa} .
$$

This is done by introducing a local coordinate system at each point on every isofrequency curve throughout the entire BZ. At fixed frequency there may exist multiple curves from multiple band surfaces, and so we denote the $n$th curve by $C_{n}$. This local coordinate system is defined about the point $\boldsymbol{\kappa}$, which has a corresponding wave number $k_{n}$, and is given by $(s, t)$, where $t$ points in the direction of the group velocity vector and $s$ is tangent to the curve. At the point $k_{n}(\boldsymbol{\kappa})$ we have

$$
\begin{aligned}
\left.k_{n}(s, t)\right|_{t=0} & =k, \\
\left.\partial_{s} k_{n}(s, t)\right|_{t=0} & =0 .
\end{aligned}
$$

We then take a Taylor series expansion near $t=0$ to obtain

$$
k-k_{n}(s, t)=k-\left\{k_{n}(s, 0)+t \partial_{t} k_{n}+s \partial_{s} k_{n}+O\left(s^{2}\right)+O\left(t^{2}\right)\right\}=-t \partial_{t} k_{n} .
$$

Accordingly (4.12) takes the form

$$
\sum_{n} \int_{-\pi / d}^{\pi / d} \int_{-\pi / d}^{\pi / d} \delta\left[k-k_{n}\left(\kappa_{x}, \kappa_{y}\right)\right] \mathrm{d} \kappa_{x} \mathrm{~d} \kappa_{y}=\sum_{n} \iint \delta\left[t \partial_{t} k_{n}\right] \mathrm{d} t \mathrm{~d} s,
$$

where one can use the well-known identity $\delta(a x)=\delta(x) /|a|$ to write

$$
\iint \frac{1}{\left|\partial_{t} k_{n}\right|} \delta(t) \mathrm{d} t \mathrm{~d} s=\int_{C_{n}} \frac{1}{\left|\partial_{t} k_{n}(s)\right|} \mathrm{d} s .
$$

Since the relationship between angular frequency and wave number is quadratic for PlaCs $\left(\omega=k^{2}\right)[29]$, using the chain rule we can determine that

$$
\mathbf{v}_{g}=\left(\partial_{\kappa_{x}} \omega, \partial_{\kappa_{y}} \omega\right)=2 k\left(\partial_{\kappa_{x}} k, \partial_{\kappa_{y}} k\right),
$$

which has an associated norm $\left|\mathbf{v}_{g}\right|$. This can be expressed in terms of the local coordinate system $(s, t)$ at the point $k_{n}(\boldsymbol{\kappa})$ to obtain

$$
\left|\mathbf{v}_{g}\right|=2 k \sqrt{\left(\partial_{\kappa_{x}} k\right)^{2}+\left(\partial_{\kappa_{y}} k\right)^{2}}=2 k\left|\partial_{t} k_{n}(s)\right| .
$$

Subsequently the DOS can be expressed in the form

$$
\mathcal{N}(k)=\frac{k}{2 \pi^{2}} \sum_{m} \int_{C_{m}} \frac{1}{\left|\mathbf{v}_{g}(s)\right|} \mathrm{d} s,
$$

where $s$ parametrizes each isofrequency contour with respect to arc length. This final representation can be evaluated numerically once the band surfaces of the crystal are known. The procedure for computing the band surfaces of a pinned plate is outlined in $[22]$. 


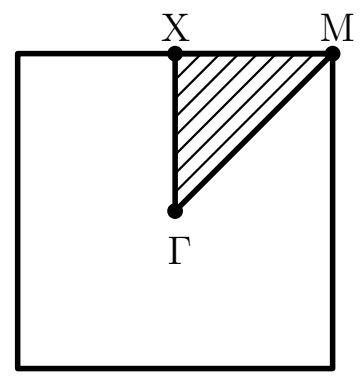

FIG. 2. An outline of the BZ for a square lattice of period d, with the symmetry points $\Gamma, \mathrm{X}$, and $\mathrm{M}$ shown. The shaded wedge denotes the irreducible BZ.

4.3. LDOS for a pinned elastic plate. As discussed earlier, we can also determine the DOS from the Green's function for a single source in a PlaC, in a vein similar to the work by $[2,3,1]$ for finite clusters. Using the method of fictitious sources, this Green's function can be expressed in the form

$$
G_{L}^{P}\left(\mathbf{x}, \mathbf{x}^{\prime} ; k\right)=G_{L}^{E}\left(\mathbf{x}, \mathbf{x}^{\prime} ; k\right)+\sum_{p} a_{m n} G_{L}^{E}\left(\mathbf{x}, \mathbf{R}_{p} ; k\right),
$$

where $G_{L}^{E}$ is the single-source Green's function in free space given in (3.13). However, we cannot impose a conventional quasi periodicity condition on (4.17) in order to reduce the number of unknowns $a_{m n}$. This is because the phase relationship from one pin to the next for a single source is much more complicated than for a plane wave or array of sources. Accordingly, deriving the Green's function for a single source in a $\mathrm{PlaC}$ is a nontrivial task, and the authors are unaware of any work in the literature which attempts to compute the Green's function for this problem directly.

However, we can obtain the LDOS by integrating the MDOS over the BZ:

$$
\mathcal{L}\left(\mathbf{x}^{\prime} ; k\right)=\frac{1}{A_{\mathrm{BZ}}} \int_{\mathrm{BZ}} \mathcal{M}\left(\mathbf{x}^{\prime} ; k, \boldsymbol{\kappa}\right) \mathrm{d} \boldsymbol{\kappa}=\frac{k}{2 \pi^{2}} \sum_{m} \int_{C_{m}}\left\{\frac{\left|\psi_{m}^{P}\left(\mathbf{x}^{\prime} ; k, \boldsymbol{\kappa}(s)\right)\right|^{2}}{\left|\mathbf{v}_{g}(s)\right|}\right\} \mathrm{d} s,
$$

where we have defined $\left|\psi_{m}^{P}\left(\mathbf{x}^{\prime} ; k, \boldsymbol{\kappa}(s)\right)\right|^{2}$ in (4.7)-(4.9).

4.4. Evaluation of LDOS over the irreducible BZ. It was shown in [36], and emphasized in [9], that in order to evaluate the LDOS over the irreducible BZ (see Figure 2), we must evaluate the Bloch mode at additional points which correspond to the set of all point group symmetry operations for the WSC geometry. For the case of a two-dimensional square lattice there are seven additional points to be considered,

$$
\mathcal{L}\left(\mathbf{x}^{\prime} ; k\right)=\frac{k}{16 \pi^{2}} \sum_{m} \int_{C_{m}(\mathrm{IBZ})}\left\{\frac{\sum_{j=1}^{8}\left|\psi_{m}^{P}\left(\boldsymbol{\beta}_{j} \mathbf{x}^{\prime} ; k, \boldsymbol{\kappa}(s)\right)\right|^{2}}{\left|\mathbf{v}_{g}(s)\right|}\right\} \mathrm{d} s,
$$

where $\boldsymbol{\beta}_{j}$ denote the point group symmetry operations. These are given by the matrices

$$
\begin{aligned}
& \boldsymbol{\beta}_{1}=\left[\begin{array}{ll}
1 & 0 \\
0 & 1
\end{array}\right], \boldsymbol{\beta}_{2}=\left[\begin{array}{rr}
1 & 0 \\
0 & -1
\end{array}\right], \boldsymbol{\beta}_{3}=\left[\begin{array}{rr}
-1 & 0 \\
0 & 1
\end{array}\right], \boldsymbol{\beta}_{4}=\left[\begin{array}{rr}
-1 & 0 \\
0 & -1
\end{array}\right] \\
& \boldsymbol{\beta}_{5}=\left[\begin{array}{ll}
0 & 1 \\
1 & 0
\end{array}\right], \boldsymbol{\beta}_{6}=\left[\begin{array}{rr}
0 & 1 \\
-1 & 0
\end{array}\right], \boldsymbol{\beta}_{7}=\left[\begin{array}{rr}
0 & -1 \\
-1 & 0
\end{array}\right], \boldsymbol{\beta}_{8}=\left[\begin{array}{rr}
0 & -1 \\
1 & 0
\end{array}\right]
\end{aligned}
$$

Copyright $@$ ( ) by SIAM. Unauthorized reproduction of this article is prohibited. 


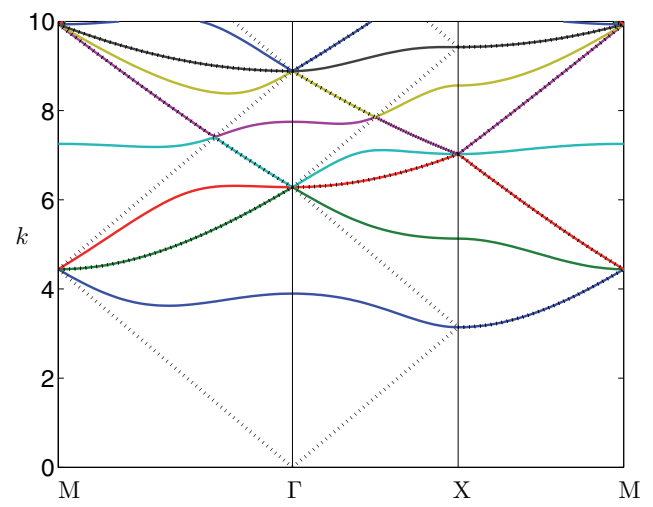

FIG. 3. Band diagram as a function of wave number $k$ for a pinned PlaC in a square lattice. The dotted lines represent light lines, and the colored lines represent band surfaces.

5. DOS for platonic clusters. Using platonic clusters (finite PlaCs), we can determine an estimate for the DOS and LDOS associated with an infinite structure straightforwardly, and we begin with the derivation of the cluster LDOS using the single-source Green's function.

For a finite cluster in two dimensions, we have $N$ pins at the coordinate points $\mathbf{x}_{n}$ with a single source located at $\mathbf{x}^{\prime}$. Accordingly, we write the Green's function for this cluster problem as

$$
G_{L}^{C}\left(\mathbf{x}, \mathbf{x}^{\prime} ; k\right)=G_{L}^{E}\left(\mathbf{x}, \mathbf{x}^{\prime} ; k\right)+\sum_{n=1}^{N} a_{n} G_{L}^{E}\left(\mathbf{x}, \mathbf{x}_{n} ; k\right),
$$

where $G_{L}^{E}$ represents the free-space Green's function defined in (3.13), which is regular when the field and source points coincide, i.e., $G_{L}^{E}\left(\mathbf{x}^{\prime}, \mathbf{x}^{\prime} ; k\right)=\mathrm{i} / 8 k^{2}$. We then impose the zero displacement boundary condition at each of the pins to obtain an $N \times N$ linear system of the form

$$
G_{L}^{E}\left(\mathbf{x}_{m}, \mathbf{x}^{\prime} ; k\right)+\sum_{n=1}^{N} a_{n} G_{L}^{E}\left(\mathbf{x}_{m}, \mathbf{x}_{n} ; k\right)=0 \quad \text { for } m=1, \ldots, N .
$$

This can be solved directly to obtain the coefficients $a_{n}$ and ultimately the Green's function for the cluster. In order to determine the LDOS for the cluster we seek the imaginary component of the Green's function $G_{L}^{C}$ as $\mathbf{x} \rightarrow \mathbf{x}^{\prime}$, which admits the form

$$
\mathcal{L}^{C}\left(\mathbf{x}^{\prime} ; k\right)=\frac{4 k^{3}}{\pi} \operatorname{Im}\left\{G_{L}^{C}\left(\mathbf{x}^{\prime}, \mathbf{x}^{\prime} ; k\right)\right\}=\frac{k}{2 \pi}+\frac{4 k^{3}}{\pi} \operatorname{Im}\left\{\sum_{n=1}^{N} a_{n} G_{L}^{E}\left(\mathbf{x}^{\prime}, \mathbf{x}_{n} ; k\right)\right\} .
$$

An estimate for the DOS can then be made via numerical integration of (5.3) over the WSC, as defined in (3.15).

6. Results. In Figure 2 an outline of the $\mathrm{BZ}$ was drawn for the case of a square array. The irreducible BZ is shaded and is enclosed by the symmetry points $\Gamma=(0,0)$, $\mathrm{X}=(\pi / d, 0)$, and $\mathrm{M}=(\pi / d, \pi / d)$. The band diagram, constructed by tracing a path around the irreducible BZ, is given in Figure 3.

Copyright (C) by SIAM. Unauthorized reproduction of this article is prohibited. 


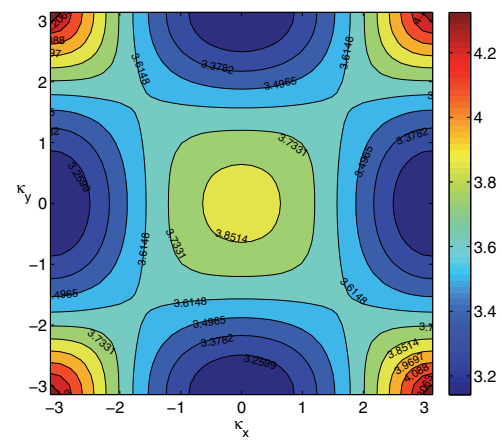

(a)

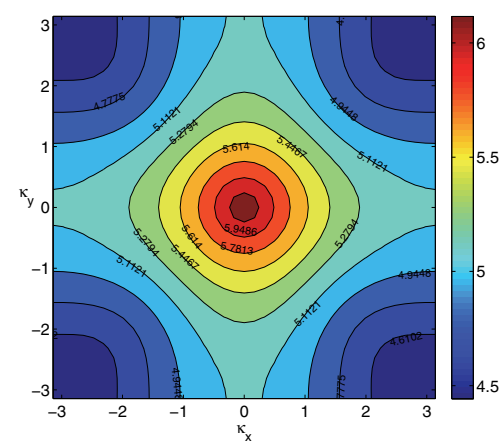

(b)

FIG. 4. The isofrequency contours of the (a) first band surface and (b) second band surface, for a square two-dimensional lattice of pins (period unity).

There is a complete band gap for $k \in(0, \pi / d)$ for a square lattice of period $d$, above which there are no further band gaps, with the exception of Dirac, or Dirac-like points, where the gap is infinitesimally thin $[22,29]$. There are many Dirac and Diraclike cones throughout the spectrum (e.g., $k(\mathrm{M})=4.4429, k(\Gamma)=2 \pi, k(\mathrm{X})=7.0248$, and $k(\Gamma)=8.8858$ ), as well as several band crossings. The light lines are denoted in the figure by dotted lines and correspond to wave numbers where the lattice sum $S_{l}^{\mathrm{Y}}$ is singular, or equivalently, $k$-values where free-space propagation (with an additional quasi periodicity condition) is supported. The equation for the light lines is given by $k=Q_{p}$. One can clearly see that there are instances where bands are sandwiched between light lines [18], giving rise to dynamic neutrality, where waves travel through the structure as if the pins were not there. The most important feature observed in Figure 3 is the threefold degeneracy seen at $k(\mathrm{M})=4.4429$.

In Figures 4(a) and 4(b) we plot the two lowest band surfaces for a square array over the entire BZ. The projection of these surfaces on the irreducible BZ forms the lowest two curves in Figure 3. These band surfaces possess strong curvature and demonstrate the highly anisotropic nature of pinned PlaCs [32, 29]. In Figure 4(a) we have a minimum value of $k(\mathrm{X})=\pi$, a saddle point of $k=3.6239$ located at $\kappa=( \pm 1.6650, \pm 1.6650)$, an absolute maximum of $k(\Gamma)=3.8961$, and an absolute maximum of $k(\mathrm{M})=4.4429$. In Figure $4(\mathrm{~b})$, the position of these critical points has changed significantly, and we have absolute minima of $k(\mathrm{M})=4.4429$, saddle points at $k(\mathrm{X})=5.1293$, and an absolute maximum of $k(\Gamma)=2 \pi$.

In Figure 5(a) we plot the DOS given by (4.16) for $k \in[3,5.5]$. There is a complete band gap for $k \leq \pi / d$ for square arrays of pins embedded in a thin plate, where $\mathcal{N}(k) \equiv 0$ by definition, and so the majority of this low-frequency band gap is omitted here. The curvature of the DOS reveals the frequencies at which critical points on band surfaces exist [9]. At a relative or absolute minimum, the local behavior of the DOS can be given by $\mathcal{N}(k)=A H(k)+B$, where $H(k)$ denotes a Heaviside function. Similarly, at a maximum point $\mathcal{N}(k)=A H(-k)+B$, and a saddle point $\mathcal{N}(k)=A \log \left|\omega-\omega_{0}\right|+B$ (where $\omega_{0}$ denotes the van Hove singularity [35]). The constants $A$ and $B$ differ between each of these expressions. From Figure 5(a) we can then determine that there is a band minimum at $k=\pi$, a saddle point at $k=3.6239$, a band maximum at $k=3.8961$, a band minimum at $k=4.4429$, and a saddle point at $k=5.1293$. One notable feature of this $\mathcal{N}(k)$ curve is an interval of suppressed DOS 


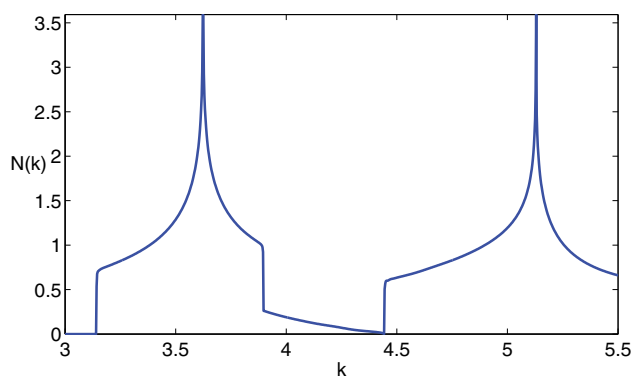

(a)

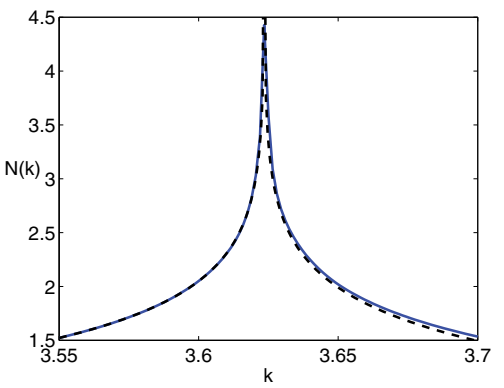

(b)

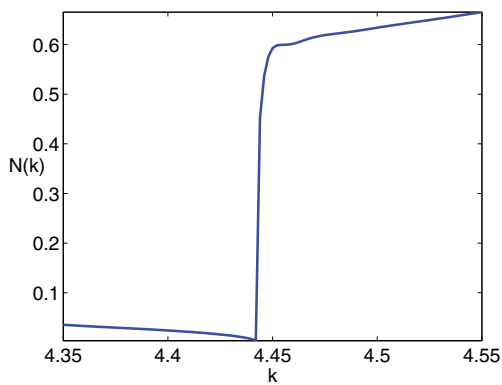

(c)

FIG. 5. The DOS for a square pinned PlaC (a) for $k \in(0,5.5]$, (b) in the vicinity of the first saddle point with the dashed asymptotic curve $\mathcal{N}_{\text {approx }}(k)$, and $(\mathrm{c})$ in the vicinity of the absolute maximum of the first band surface and minimum of the second and third band surfaces at $k=4.4429$.

for $k \in[3.8961,4.4429]$. This interval of naturally suppressed DOS corresponds to strong energy localization (as will be shown in Figures 7 and 11 for a finite cluster). In this interval of suppressed DOS, the square array of pins diminishes the power radiated by a source in the lattice.

A close-up of the DOS near $k=4.4429$ is given in Figure 5(c), where the DOS curve does not progress to zero linearly. Therefore this is not a Dirac point degeneracy, which requires the bands above and below to collapse in the shape of cones [30]. Another feature of this image is the small oscillations in the DOS after the point $k=4.4429$. This is not a numerical error, but a feature of the varying group velocities which are present at the threefold degeneracy seen here. There are a host of behaviors associated with Dirac and Dirac-like points, including resonant spikes in transmission, which are ongoing topics of investigation. In Figure 5(b) the DOS near the first saddle point is drawn, with the asymptotic curve $\mathcal{N}_{\text {approx }}$ overlaid, where the DOS is infinite at the saddle point. For $k=3.6239$ the critical point values are given by $A=-0.4660$ and $B=1.2231$. Using this $A$ parameter, one can then determine the leading order behavior of the level shift near this point (see Table 1 in [9]).

The DOS for a square array of pins is drawn over a higher range $k \in[6.2,8.7]$ in Figure 6, where van Hove singularities are observed at $k=6.3125, k=7.1138, k=$ $7.1845, k=7.7056, k=8.3849$, and $k=8.5635$; band minima at $k=2 \pi, k=7.0248$ (corresponding to the Dirac-like frequency), $k=7.4319$, and $k=7.8838$; and band maxima at $k=7.2539, k=7.3758, k=7.7484$, and $k=7.8245$. This plot shows 


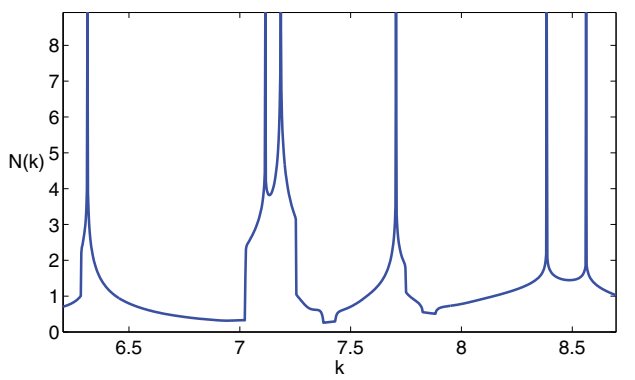

FIG. 6. The DOS for a square pinned PlaC from $k \in[6.2,8.7]$.

intervals of suppressed DOS for $k \in[7.3758,7.4319]$ and $k \in[7.8245,7.8838]$. These intervals are not as strongly suppressed as $k \in[3.8961,4.4429]$, and this is validated in Figure 7, where the displacement for a $13 \times 13$ finite cluster is calculated for a source located at $\mathbf{x}^{\prime}=(1 / 2,1 / 2)$. In Figure $7(\mathrm{a})$ very strong localization of energy is observed at $k=4.1$ with $\max |\operatorname{Re}(u)|=16 \times 10^{-3}$, relative to the other figures here. Figures 7 (c) and 7 (e) ( $k=7.4$ and $k=7.85$, respectively) also display strong localization of energy. The most coherent leakage of energy is seen in Figure 7(e), which forms a St. George's Cross through the cluster and into the plate. The isofrequency contours (Figures 7(b), $7(\mathrm{~d})$, and $7(\mathrm{f})$ ) corresponding to the array problem are drawn for comparison, where the central box denotes the edges of the BZ.

Recall that the LDOS is a function of wave number $k$ and source location $\mathbf{x}^{\prime}$ and is a measure of the local availability of band surfaces to which a source can couple and radiate power through an array. The DOS is the spatial average of the LDOS over the fundamental cell. In Figure 8 we plot the LDOS for a selection of values where one can see that, for fixed frequency, the location of the source changes the power radiated into the array significantly. This makes sense when we consider the fact that intervals of vanishing DOS correspond to intervals of vanishing LDOS [8]. However, the reverse does not hold in general, as the Bloch mode of a crystal may have symmetry points where the mode vanishes (i.e., nodes), and so the LDOS can differ considerably based on the location of the source. In Figure 8(a) we compute the LDOS for $k=3.4$, which has a maximum value of $\mathcal{L}=2.0949$ at $\mathbf{x}^{\prime}=( \pm 1 / 2, \pm 1 / 2)$, a saddle point value of $\mathcal{L}=1.0309$ at $\mathbf{x}^{\prime}=( \pm 0, \pm 1 / 2)$ and $\mathbf{x}^{\prime}=( \pm 1 / 2, \pm 0)$, and a minimum of $\mathcal{L}=0$ at the origin. For the pinned plate, the location of the minimum remains constant for all $k$, as it coincides with the location of the central pin, where the displacement is specified to be zero. If one integrates this LDOS surface over the WSC, we can obtain $\mathcal{N}(k)=1.0314$, which matches the value given in Figure 5 (a). The LDOS surfaces in the vicinity of the saddle point $k=3.6239$, and near the absolute maximum $k=3.8961$, exhibit curvature identical to that of Figure 8(a) but with different ranges of values (whose scale can be deduced by examining the DOS curve shown previously).

The LDOS surface for $k=4.6$ is drawn in Figure 8(b), with a lower maximum value of $\mathcal{L}=1.3594$ relative to the LDOS surface for $k=3.4$. The location of the maximum LDOS has now shifted to $\mathbf{x}^{\prime}=(0, \pm 1 / 2)$ and $\mathbf{x}^{\prime}=( \pm 1 / 2,0)$, and points of zero LDOS are now located at $\mathbf{x}^{\prime}=( \pm 1 / 2, \pm 1 / 2)$ in addition to the origin. There is also a saddle point of $\mathcal{L}=0.7020$ at $\mathbf{x}^{\prime}=( \pm 0.2590, \pm 0.2590)$. This LDOS function integrates to a DOS value of $\mathcal{N}(k)=0.7009$. In Figure 8(c) we draw the contour plot 


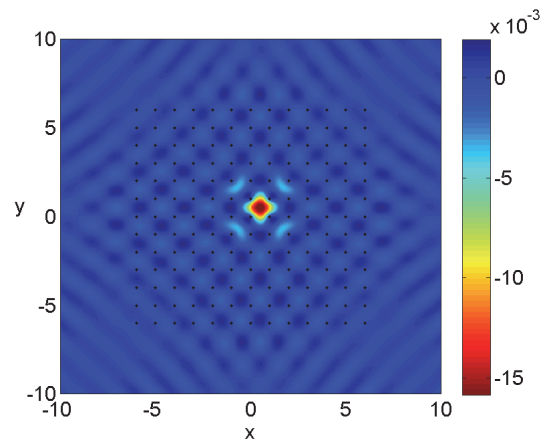

(a)

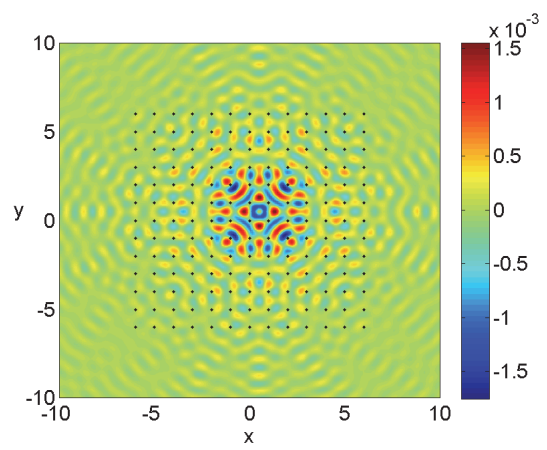

(c)

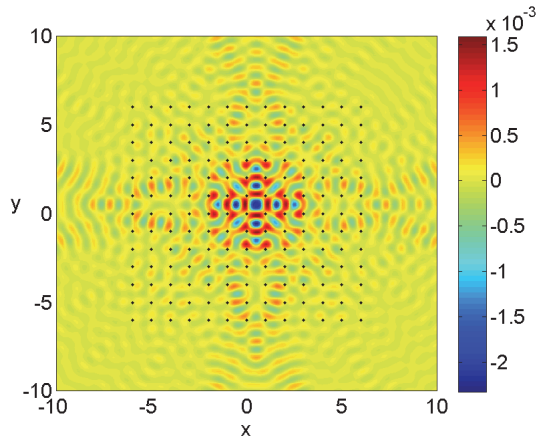

(e)

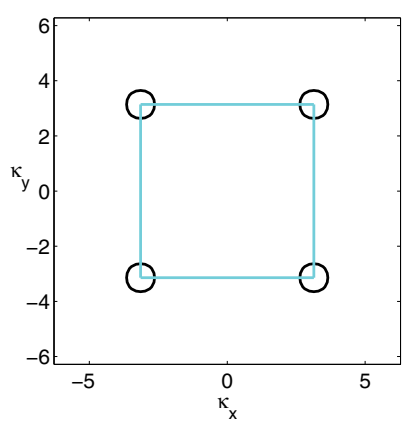

(b)

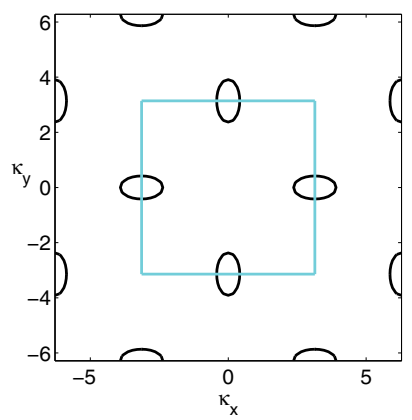

(d)

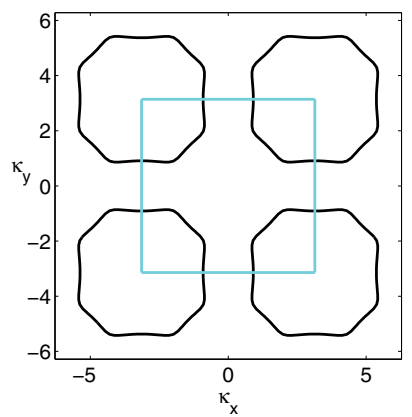

(f)

FIG. 7. The displacement fields $\operatorname{Re}(u)$ for a $13 \times 13$ finite cluster, where $\mathbf{x}^{\prime}=(1 / 2,1 / 2)$ and (a) $k=4.1$, (c) $k=7.4$, and (e) $k=7.85$. Also shown are the associated isofrequency contours in (b), (d), and (f), respectively, where the box denotes the outline of the BZ.

for the LDOS surface corresponding to $k=5.6$. This features a maximum value of $\mathcal{L}=0.9245$ at $\mathbf{x}^{\prime}=( \pm 1 / 2,0)$ and $\mathbf{x}^{\prime}=(0, \pm 1 / 2)$, a relative minimum of $\mathcal{L}=0.5885$ in the furthest corners of the WSC, and an absolute maximum at the origin of zero. There are also four saddle points located at $\mathbf{x}^{\prime}=( \pm 0.3283, \pm 0.3283)$ for $\mathcal{L}=0.6866$. The integral of this LDOS surface over the fundamental cell gives $\mathcal{N}=0.6075$. The contours of the LDOS at $k=6.6$ are drawn in Figure 8(d), where there is a significant change in the qualitative behavior of the LDOS surface, relative to the previous 


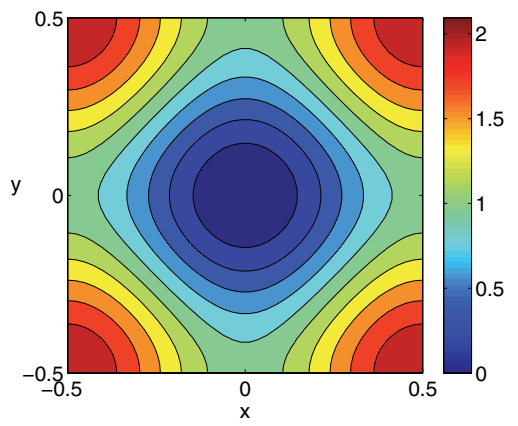

(a)

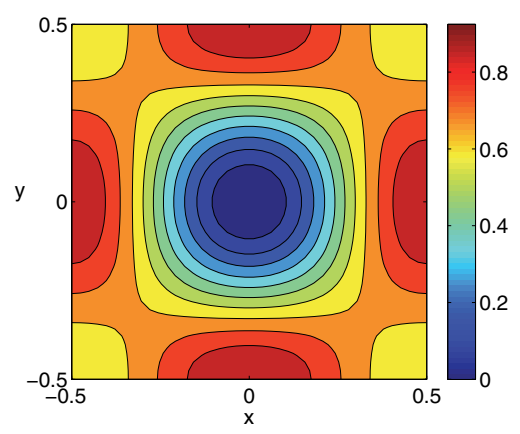

(c)

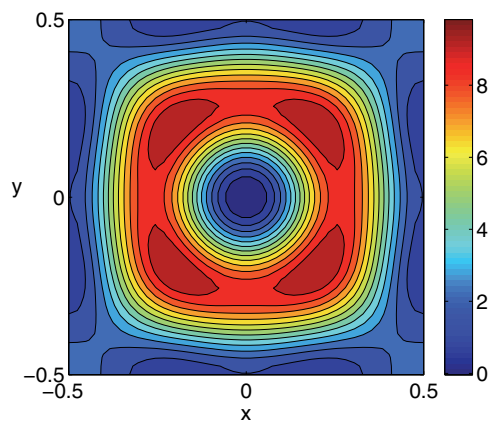

(e)

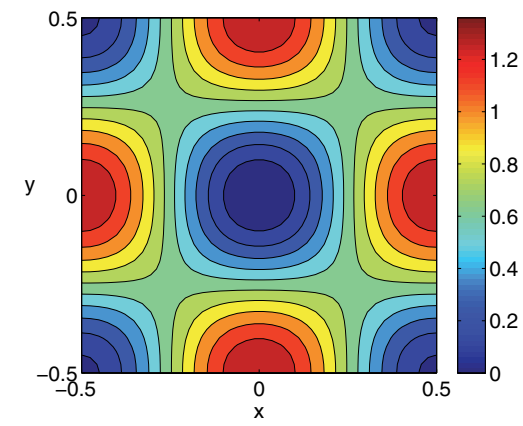

(b)

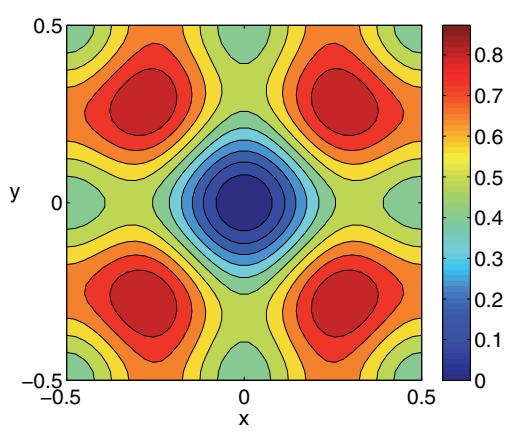

(d)

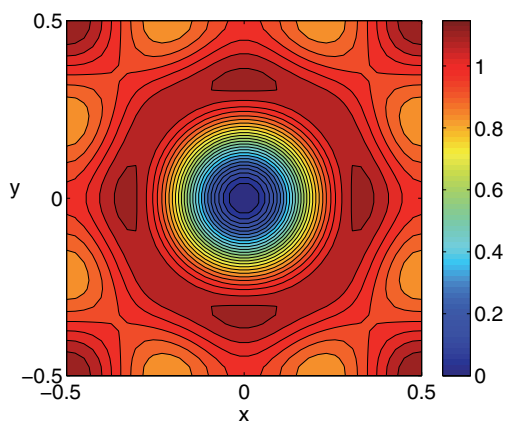

(f)

FIG. 8. LDOS generated over the WSC for (a) $k=3.4$, (b) $k=4.6$, (c) $k=5.6$, (d) $k=6.6$, (e) $k=7.2$, and (f) $k=8.1$.

pictures. Here the maximum LDOS $(\mathcal{L}=0.8833)$ is located at $\mathbf{x}^{\prime}=( \pm 5 / 18, \pm 5 / 18)$. There is one minimum point at the origin, and there are now eight relative minimum points at $(0, \pm 1 / 2),( \pm 1 / 2,0)$, and $( \pm 1 / 2, \pm 1 / 2)$ corresponding to $\mathcal{L}=0.4202$. There are four saddle points at $(0, \pm 0.3182)$ and $( \pm 0.3182,0)$ corresponding to $\mathcal{L}=0.5178$, and the integral of LDOS over the WSC at $k=6.6$ gives a DOS value of $\mathcal{N}=0.5887$.

The LDOS is drawn over the WSC for $k=7.2$ in Figure 8(e), where we observe an absolute maximum of $\mathcal{L}=9.8257$ at $\mathbf{x}^{\prime}=( \pm 0.2020, \pm 0.2020)$, a relative maximum of 


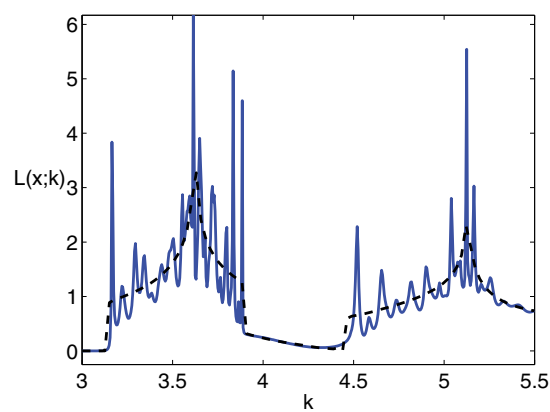

(a)

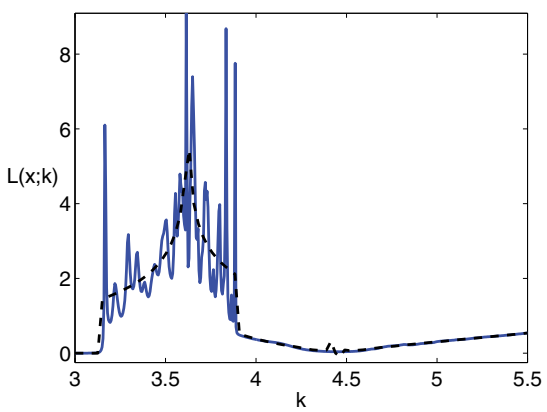

(b)

FIG. 9. The LDOS for a $13 \times 13$ finite cluster at (a) $\mathbf{x}^{\prime}=(1 / 4,1 / 3)$ and (b) $\mathbf{x}^{\prime}=(1 / 2,1 / 2)$. The dotted black line denotes the LDOS for an infinite PlaC.

$\mathcal{L}=8.8870$ at $\mathbf{x}^{\prime}=( \pm 0.2677,0)$ and $\mathbf{x}^{\prime}=(0, \pm 0.2677)$, a saddle point of $\mathcal{L}=2.1711$ at $\mathbf{x}^{\prime}=( \pm 0.4192, \pm 0.4192)$, and an absolute minimum of zero at the origin. There are also critical points of $\mathcal{L}=2.5006$ at $\mathbf{x}^{\prime}=( \pm 1 / 2, \pm 1 / 2), \mathcal{L}=1.3906$ at $\mathbf{x}^{\prime}=(0, \pm 1 / 2)$ and $\mathbf{x}^{\prime}=( \pm 1 / 2,0)$, and $\mathcal{L}=0.8249$ at $\mathbf{x}^{\prime}=( \pm 0.2071, \pm 0.5)$ and $\mathbf{x}^{\prime}=( \pm 0.5, \pm 0.2071)$. A large maximum $\mathcal{L}$ value is observed here and coincides with an interval of enhanced DOS possessing two saddle points, as seen in Figure 6. The integral of this surface over the WSC gives a value of $\mathcal{N}=4.8558$. In Figure $8(\mathrm{f})$ the LDOS is drawn for $k=8.1$, which has an absolute maximum of $\mathcal{L}=1.1461$ at $\mathbf{x}^{\prime}=( \pm 1 / 2, \pm 1 / 2)$. There are also saddle points at $( \pm 0.3535, \pm 0.3535)$, as well as at $(0, \pm 1 / 2)$ and $( \pm 1 / 2,0)$ for $\mathcal{L}=0.9697$ and $\mathcal{L}=0.9852$, respectively. There is a relative maximum located at $( \pm 1 / 3,0)$ and $(0, \pm 1 / 3)(\mathcal{L}=1.1191)$, a relative minimum at $( \pm 0.2172, \pm 1 / 2)$ and $( \pm 1 / 2, \pm 0.2172)(\mathcal{L}=0.8470)$, and a point of zero LDOS at the origin. Here the integral of LDOS over the WSC gives a value of $\mathcal{N}=0.9281$.

The LDOS for a $13 \times 13$ finite cluster is drawn at two source locations in Figure 9, based on the expression (5.3). In Figure 9(a) the source is located at $\mathbf{x}^{\prime}=(1 / 4,1 / 3)$, away from a line of symmetry. Also drawn is the LDOS for an infinite pinned crystal. From this we can observe that the LDOS for a finite cluster is a superposition of the LDOS for an infinite crystal plus the resonances for a cluster. These resonances are all of different strength and depend on how close the imaginary part of the resonance sits to the real line (note that these resonances are referred to as complex scattering frequencies in [20]). It is difficult to establish the strength of the resonances accurately, due to the effects of finite sampling in $k$. In Figure 9 (b) we plot the LDOS for a $13 \times 13$ finite cluster with the source point positioned at $\mathbf{x}^{\prime}=(1 / 2,1 / 2)$. This choice of source location corresponds to strong field localization analogous to that in Figure 7(a), but over the wider interval $k \in[3.8961,5.5]$ (the range considered here). Also featured in this plot is the LDOS for an infinite structure, which shows a small oscillation at $k=4.4429$, corresponding to the triple point degeneracy at M.

For the $13 \times 13$ cluster, the widths of the peaks caused by the cluster resonances appear approximately similar for both source locations over the first band surface. We find that as cluster sizes increase, the distribution of spikes becomes denser and the peaks become sharper, as shown in Figure 10, where we compute the DOS for a $21 \times 21$ cluster. This curve is computed by evaluating (5.3) and numerically integrating over the WSC. In Figure 11 we consider the $\log |\operatorname{Re}(u)|$ field for an $11 \times 11$ finite cluster with 


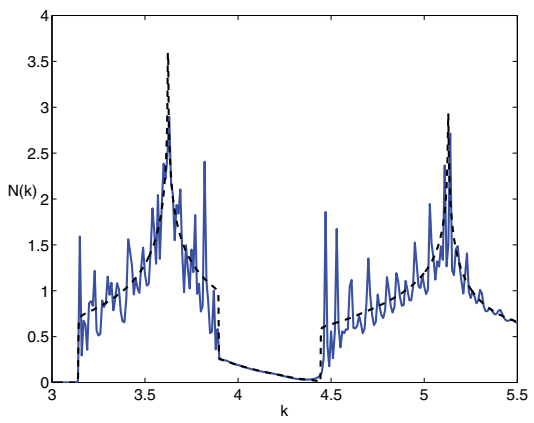

FIG. 10. The DOS curve generated for a $21 \times 21$ finite cluster. The dotted black line represents the DOS for an infinite PlaC.

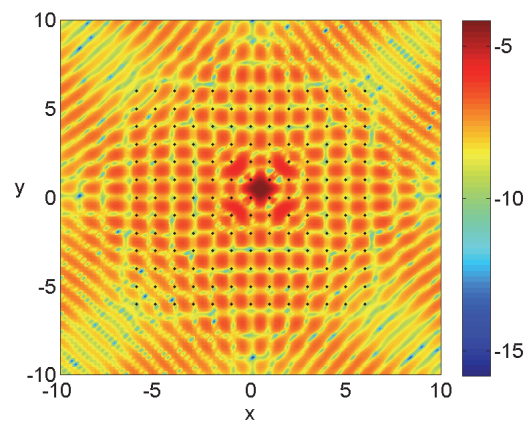

(a)

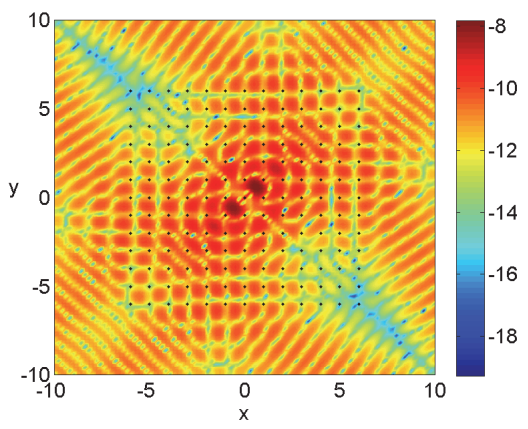

(b)

FIG. 11. The displacement field $\log |\operatorname{Re}(u)|$ for a finite cluster at $k=4.1$, where (a) $\mathbf{x}^{\prime}=$ $(1 / 2,1 / 2)$ and $(\mathrm{b}) \mathbf{x}^{\prime}=(0.01,0.01)$.

a single source. In Figure 11(a) the source is located at $\mathbf{x}^{\prime}=(1 / 2,1 / 2)$ and exhibits a monopole-style localization of energy, or field confinement, in the vicinity of the source location. We also observe that the principal direction of energy propagation is along the lines $y=x$ and $y=-x$, outgoing in both directions. This St. Andrew's Cross emission pattern is best observed outside the cluster, and the directions coincide with the isofrequency contours in Figure $4(\mathrm{a})$ at $k=4.1$. If one considers a larger finite cluster, the amplitude of the peak at $\mathbf{x}=(1 / 2,1 / 2)$ does not grow, and so this behavior does not correspond to a resonance. As the source point approaches the pin along the symmetry line $y=x$, as seen in Figure 11(b) (where the source is located at $\left.\mathbf{x}^{\prime}=(0.01,0.01)\right)$, we observe a trough of minimal displacement along $y=-x$ and emission of energy into the plate along $y=x$ in both directions. It is shown in [1] that square clusters exhibit strong leakage at the corners, and so this may be a consequence of the finite square geometry; however, investigations into other finite geometries have been made, and these all exhibit the same St. Andrew's cross pattern in the displacement.

7. Concluding remarks. Using the Green's function method [17], we have computed the MDOS, SDOS, LDOS, and DOS functions for a pinned platonic crystal, a finite cluster of pins, and for an empty lattice. It is shown that pinned plates feature wave number intervals where the DOS and LDOS are suppressed by the square array, 
which corresponds to nonresonant field concentration in finite PlaCs. It is also shown that the computation of the DOS and LDOS for finite clusters can isolate these localization regions successfully. This is the first known investigation into the DOS for platonic crystals and provides a general foundation for the investigation of other structured plates. These include different pinned array geometries (i.e., hexagonal lattices of pins), and perforated plates subject to clamped- and free-edge conditions, following from $[22,28]$.

\section{REFERENCES}

[1] A. A. Asatryan, L. C. Botten, N. A. Nicorovici, R. C. McPhedran, and C. M. de Sterke, Frequency shift of sources embedded in finite two-dimensional photonic clusters, Waves Random Complex Media, 16 (2006), pp. 151-165.

[2] A. A. Asatryan, K. Busch, R. C. McPhedran, L. C. Botten, C. M. de Sterke, and N. A. NicorovicI, Two-dimensional Green's function and local density of states in photonic crystals consisting of a finite number of cylinders of infinite length, Phys. Rev. E, 63 (2001), 046612.

[3] A. A. Asatryan, S. Fabre, K. Busch, R. C. McPhedran, L. C. Botten, C. M. de Sterke, AND N. A. NicorovicI, Two-dimensional local density of states in two-dimensional photonic crystals, Opt. Express, 8 (2001), pp. 191-196.

[4] M. Brun, A. B. Movchan, I. S. Jones, and R. C. McPhedran, Bypassing shake, rattle and roll, Phys. World, 26 (2013), pp. 32-36.

[5] S. K. Chin, N. A. Nicorovici, and R. C. McPhedran, Green's function and lattice sums for electromagnetic scattering by a square array of cylinders, Phys. Rev. E, 49 (1994), pp. $4590-4602$.

[6] D.V. Evans And R. Porter, Penetration of flexural waves through a periodically constrained thin elastic plate in vacuo and floating on water, J. Engrg. Math., 58 (2007), pp. 317-337.

[7] M. Farhat, S. Guenneau, and S. Enoch, Ultrabroadband elastic cloaking in thin plates, Phys. Rev. Lett., 103 (2009), 024301.

[8] D. P. Fussell, R. C. McPhedran, and C. M. De Sterke, Three-dimensional Green's tensor, local density of states, and spontaneous emission in finite two-dimensional photonic crystals composed of cylinders, Phys. Rev. E, 70 (2004), 066608.

[9] D. P. Fussell, R. C. McPhedran, And C. M. De Sterke, Two-dimensional treatment of the level shift and decay rate in photonic crystals, Phys. Rev. E, 72 (2005), 046605.

[10] D. P. Fussell, R. C. McPhedran, C. M. De Sterke, and A. A. Asatryan, Threedimensional local density of states in a finite two-dimensional photonic crystal composed of cylinders, Phys. Rev. E, 67 (2003), 045601.

[11] S. G. Haslinger, R. C. McPhedran, N. V. Movchan, and A. B. Movchan, Localisation near defects and filtering of flexural waves in structured plates, Int. J. Fracture, 184 (2013), pp. $1-17$.

[12] J. D. Joannopoulos, Photonic Crystals: Molding the Flow of Light, Princeton University Press, Princeton, NJ, 2008.

[13] A. LagendiJk and B. A. Van Tiggelen, Resonant multiple scattering of light, Phys. Rep., 270 (1996), pp. 143-215.

[14] Z. Y. LI, L. L. Lin, AND Z. Q. Zhang, Spontaneous emission from photonic crystals: Full vectorial calculations, Phys. Rev. Lett., 84 (2000), pp. 4341-4344.

[15] P. Markos and C. M. Soukoulis, Wave Propagation: From Electrons to Photonic Crystals and Left-handed Materials, Princeton University Press, Princeton, NJ, 2008.

[16] P. A. Martin, Discrete scattering theory: Green's function for a square lattice, Wave Motion, 43 (2006), pp. 619-629.

[17] R. C. McPhedran, L. C. Botten, J. McOrist, A. A. Asatryan, M. C. de Sterke, and N. A. Nicorovici, Density of states functions for photonic crystals, Phys. Rev. E, 69 (2004), 016609.

[18] R. C. McPhedran, A. B. Movchan, and N. V. Movchan, Platonic crystals: Bloch bands, neutrality and defects, Mech. Mater., 41 (2009), pp. 356-363.

[19] D. B. Melrose and R. C. McPhedran, Electromagnetic Processes in Dispersive Media, Cambridge University Press, Cambridge, UK, 2005.

[20] M. H. Meylan and R. C. McPhedran, Fast and slow interaction of elastic waves with platonic clusters, Proc. R. Soc. Lond. Ser. A Math. Phys. Eng. Sci., 467 (2011), pp. 3509-3529.

[21] A. Moroz, Minima and maxima of the local density of states for one-dimensional periodic systems, Europhys. Lett., 46 (1999), pp. 419-424. 
[22] A. B. Movchan, N. V. Movchan, And R. C. McPhedran, Bloch-Floquet bending waves in perforated thin plates, Proc. R. Soc. Lond. Ser. A Math. Phys. Eng. Sci., 463 (2007), pp. 2505-2518.

[23] A. B. Movchan, N. V. Movchan, and C. G. Poulton, Asymptotic Models of Fields in Dilute and Densely Packed Composites, Imperial College Press, London, 2002.

[24] N. I. Muskhelishvili, Singular Integral Equations: Boundary Problems of Function Theory and Their Application to Mathematical Physics, Dover, New York, 2008.

[25] S. J. Myers, D. P. Fussell, J. M. Dawes, E. MÄgi, R. C. McPhedran, B. J. Eggleton, AND C. M. DE STERKe, Manipulation of spontaneous emission in a tapered photonic crystal fibre, Opt. Express, 14 (2006), pp. 12439-12444.

[26] A. N. Norris And C. Vemula, Scattering of flexural waves on thin plates, J. Sound. Vibration, 181 (1995), pp. 115-125.

[27] C. G. Poulton, A. B. Movchan, N. V. Movchan, and R. C. McPhedran, Analytic theory of defects in periodically structured elastic plates, Proc. R. Soc. Lond. Ser. A Math. Phys. Eng. Sci., 468 (2012), pp. 1196-1216.

[28] M. J. A. Smith, M. H. Meylan, and R. C. McPhedran, Flexural wave filtering and platonic polarizers in thin elastic plates, Quart. J. Mech. Appl. Math., 66 (2013), pp. 437-463.

[29] M. J. A. Smith, R. C. McPhedran, C. G. Poulton, and M. H. Meylan, Negative refraction and dispersion phenomena in platonic clusters, Waves Random Complex Media, 22 (2012), pp. $435-458$.

[30] M. J. A. Smith, M. H. Meylan, and R. C. McPhedran, Double Dirac cones at $\kappa=0$ in pinned platonic crystals, Waves Random Complex Media, 24 (2014), pp. 35-54.

[31] M. J. A. Smith, R. Porter, and T. D. Williams, The effect on bending waves by defects in pinned elastic plates, J. Sound. Vibration, 331 (2012), pp. 5087-5106.

[32] M. J. Steel, R. Zoli, C. Grillet, R. C. McPhedran, C. M. de Sterke, A. Norton, P. BAssi, And B. J. EgGleton, Analytic properties of photonic crystal superprism parameters, Phys. Rev. E, 71 (2005), 056608.

[33] N. Stenger, M. Wilhelm, And M. Wegener, Experiments on elastic cloaking in thin plates, Phys. Rev. Lett., 108 (2012), 014301.

[34] A. Taflove, S. G. Johnson, and A. Oskooi, Advances in FDTD Computational Electrodynamics: Photonics and Nanotechnology, Artech House, Boston, 2013.

[35] L. VAN Hove, The occurrence of singularities in the elastic frequency distribution of a crystal, Phys. Rev., 89 (1953), pp. 1189-1193.

[36] R. WANG, X. WANG, B. GU, AND G. YANG, Local density of states in three-dimensional photonic crystals: Calculation and enhancement effects, Phys. Rev. B, 67 (2003), 155114.

[37] S. Wilcox, L. C. Botten, R. C. McPhedran, C. G. Poulton, and C. M. de Sterke, Modeling of defect modes in photonic crystals using the fictitious source superposition method, Phys. Rev. E, 71 (2005), 056606.

[38] M. Wubs AND A. LAGENDIJK, Local optical density of states in finite crystals of plane scatterers, Phys. Rev. E, 65 (2002), 046612.

Copyright (c) by SIAM. Unauthorized reproduction of this article is prohibited. 